\title{
3 Research Square

\section{Renal pelvis metastasis from lung cancer with extension of renal venous thrombus: a case report and literature review}

\section{Shengjiang Bai}

Sichuan University West China Hospital

Li Lai

Sichuan University West China Hospital

\section{Ming Shi}

Sichuan University West China Hospital

\section{Hang Xu}

Sichuan University West China Hospital

\section{Haoran Lei}

Sichuan University West China Hospital

\section{Weitao Zheng}

Sichuan University West China Hospital

\section{Lu Yang ( $\nabla$ wycleflue@163.com )}

Sichuan University West China Hospital

\section{Qiang Wei}

Sichuan University West China Hospital

\section{Case report}

Keywords: Lung cancer, EGFR mutation, Metastasis, Renal pelvic, Renal venous thrombus, Case report.

Posted Date: August 11th, 2020

DOI: https://doi.org/10.21203/rs.3.rs-56047/v1

License: (c) (1) This work is licensed under a Creative Commons Attribution 4.0 International License. Read Full License 


\section{Abstract}

Background Non-Small Cell Lung Cancer (NSCLC) rarely metastases to the renal pelvis, especially with renal venous tumor thrombus, we presented the first case of a NSCLC patient with renal pelvis metastasis and renal venous invasion.

Case presentation A 50-year-old man was referred to the thoracic facility after experiencing a healthy examination revealing solitary pulmonary mass in the left lung, he received surgery of left upper lobe resection and hilar and mediastinal lymph nodes dissection at our hospital in June 2016. Postoperative histopathologic diagnosis was NSCLC (pT3NOM0), and regular follow-up was performed after operation. After eighteen months, he was referred to the urology facility complaining of visible hematuria and pain in the right waist. Abdominal enhanced computed tomography (CT) indicated a neoplasm in the right renal pelvis, and filling coloboma could be seen in the right renal vein during the CT enhancement period. Simultaneously two nodules were found in his right lung. He received radical nephrectomy (RN) for the right kidney and removal of renal venous thrombus in December 2017. The neoplasm occupying the right pelvis and the solid ingredient occupying the right renal venous lumen were histologically considered as a metastatic carcinoma from the NSCLC. Icotinib Hydrochloride Tablets were adminstrated to treat nodules in the right lung since the RN. According to the last re-examination in March 2020, the efficacy was evaluated as partial remission.

Conclusions Renal venous invasion of renal tumor not only occurs in renal cell carcinoma (RCC) and upper urinary tract urothelial carcinoma (UTUC), but also can be seen in the state of metastasis. Acquiring correct preoperative diagnosis is of great importance in determining proper treatment strategies. Besides, the aggressive RN for patients suffering advanced metastatic carcinoma of renal pelvis is safe under perfect perioperative management and favorable to treat metastatic carcinoma in other sites.

\section{Background}

Despite remarkable progress has been achieved in medical research over the last decades, lung cancer is still the most common cancer type worldwide and the leading cause of cancer-related deaths [1.2], with the World Health Organization estimating 1.37 million deaths globally per year [3]. NSCLC accounts for $88 \%$ of all diagnosis of lung cancer [4] and it could almost metastasis to every site. Liver, adrenals, bones, brain and kidney are the frequently extrathoracic sites, but renal pelvis metastasis from lung cancer with renal venous thrombus is extremely rare, here we presented a precious and interesting case for diagnosis and therapy of advanced NSCLC.

\section{Case Presentation}

A 50-year-old man with smoking history was referred to our thoracic facility after undergoing a healthy examination revealing solitary pulmonary mass in the left lung without cough, hemoptysis, dyspnea, 
chest pain, or other pulmonary symptoms. His physical examination was negative. He then received thoroughly examination at our hospital. Fiberoptic bronchoscope brushing cells and liquid based cytology discovered cancer cells (non-small cell carcinoma), and the fiberoptic bronchoscopy biopsy also discovered the cancer cells. The CT scan of his chest revealed a mass in the upper left lung (Fig. 1). Examinations including TC-99 MDP bone scintigraphy, the brain enhanced CT and enhanced upper abdominal CT were all negative for distant metastasis. Consequently, he received surgery of left upper lobe resection and hilar and mediastinal lymph node dissection at West China Hospital in June 2016. Intraoperative macroscopic examination of the sample revealed a mass 6.0 centimeters in diameter, and postoperative histopathologic examination of the specimen confirmed as lung adenocarcinoma (a type of NSCLC, classified between poorly to moderately differentiated, Fig. 2). The immunohistochemistry staining indicated the expression of TTF- 1 and CK7 were positive, while the expressions of P63, CK5/6, Syn and CgA were negative (Fig. 2). Epidermal growth factor receptor (EGFR) mutated according to the gene test, without nearby lymph node metastasis. Though the pathologic stage of the patient was $\triangle \mathrm{B}$ (pT3NOMO), he was not given antitumor adjuvant therapy because of suffering pulmonary tuberculosis and viral hepatitis type B and receiving medical treatment for them. There was no local recurrence or systemic metastasis during postoperative follow-up for eighteen months.

Eighteen months after operation, the man was referred to the urology facility complaining of pain in the right waist, lower abdomen and groin area, together with visible hematuria throughout. Abdominal enhanced CT showed a neoplasm in the right renal pelvis, and filling coloboma could be seen in the right renal vein during the $\mathrm{CT}$ enhancement period. We observed an enlarged lymph node behind the inferior vena cava simultaneously (Fig. 3). Meanwhile, enhanced CT scan of his chest showed two nodules in his right lung (located in the upper lobe and the inferior lobe of the right lung), which tended to be metastatic tumors derived from his left lung (Fig. 4A and B). Vascular ultrasound detected solid ingredient occupying the right renal venous lumen. What's interesting was that the urinary cytology and Fluorescence In Situ Hybridization (FISH) were negative. The biopsy of the neoplasm in the right renal pelvis was not performed in case of great risk, we directly conducted RN for the right kidney and removal of renal venous thrombus in December 2017. The postoperative specimen of RN was histologically considered to be a metastatic carcinoma from the NSCLC but not UTUC, with immunohistochemistry stain positive for TTF1, NapsinA and focally positive for P63 and CK5/6, while negative for PAX-8, Uroplakin-2, Uroplakin-3, Syn and CgA (Fig. 5). Gene test detected 19-Del mutation of EGFR. Pathologists observed that the carcinoma had invaded the muscular layer of renal pelvis and renal parenchyma. Metastasis was found in one lymph node inside the right renal pedicle. Moreover, the solid ingredient occupying the right renal venous lumen was confirmed as agminated cancer cells.

Icotinib Hydrochloride Tablets, the preferred targeted therapy for metastatic NSCLC with EGFR mutation, were prescribed to him since the RN for the right kidney. Postoperative TC-99 MDP bone scintigraphy was negative one year later. The CT scan of chest showed nodal diameter decreased from 10 millimeter to 3.7 millimeter, in the nodule locating in the right inferior lobe according to the most recent reexamination in March 2020. Likewise, the nodule locating in the upper lobe of right lung was cured. The drug efficacy was evaluated as partial remission (Fig. 4C). 


\section{Discussion}

RCC has the ability to locally invade and progress as a tumor thrombus in the venous system, the incidence it metastases to the renal vein or inferior vena cava is 4 to $36 \%$ [5.6] and the incidence of UTUC with the renal vein or inferior vena cava invasion was reported to be 5 to $7 \%$ [7.8]. To the best of our knowledge, this is the first case that reported renal pelvis tumor metastasis from lung carcinoma extending to renal vein. It alarms that correct preoperative diagnosis of a renal tumor with renal vein tumor thrombus is crucial before surgical resection was decided.

However, differential diagnosis of the three cases remains challenge. Urinary cytology seems difficult to distinguish UTUC and metastatic carcinoma of renal pelvis owing to cytology is less sensitive for UTUC (sensitivity and positive predictive value of $56 \%$ and $54 \%$ for high-grade disease, respectively) than bladder tumors (positive predictive value $>85 \%$ for high-grade disease). Although cytology could be performed in situ in the renal cavities via ureteroscopy which is more sensitive than cytology (sensitivity and positive predictive value of $71 \%$ and $53 \%$ for detecting high-grade disease, respectively) [9], while the doctors' operation during ureteroscopy might bring out tumor metastasis.

E.Voulgaris et al. [10] performed the urinary cytology in patients with lung carcinoma without obvious urothelial metastases, which might be helpful in the early diagnosis of urothelial metastases. However, their studies seemed to included patients with metastatic/extensive disease and high tumor burden, whether urinary cytology should be performed in lung cancer needs further investigations.

Moreover, Computed tomography urography (CTU) is a powerful tool that enables detailed anatomic evaluation of the urinary tract when distinguishing UTUC, benign urinary tract conditions and infrequent metastatic tumors. It could provide additional imaging information but not conclusive evidence for determining treatment strategy. By contrast, flexible ureteroscopy biopsy can determine tumor grade in $90 \%$ of UTUC with low false-negative rate regardless of the sample size [11], and can be helpful in the decision-making process between radical nephroureterectomy (RNU) for UTUC and RN for metastatic carcinoma of renal pelvis. However, it could be done only with careful weighted benefit and disadvantage such as implantation metastasis, especially in advanced metastatic carcinoma of renal pelvis as presented here. Image enhancement techniques including Narrow band imaging, Image1 S, and Photodynamic diagnosis-guided endoscopy aim at achieving better visualization of malignant urothelium and especially flat tumors or carcinoma in situ [12], which might be helpful for differential diagnosis between UTUC and earlier metastatic tumor in renal pelvis, but the clinical value has yet to be demonstrated.

\section{Conclusions}

Renal venous invasion of renal tumor not only occur in RCC and UTUC, but also in metastatic carcinoma of renal pelvis. Differentiating these three kinds of carcinomas so as to acquire correct preoperative diagnosis is critical for determining proper treatment strategies, which should arise urological surgeons' 
attention, especially when encountering patients with the history of carcinoma in other sites. On the other hand, the aggressive RN for patients suffering advanced metastatic carcinoma of renal pelvis is safe under perfect perioperative management and favorable to treat metastatic carcinoma in other sites.

\section{Abbreviations}

NSCLC

Non-Small Cell Lung Cancer.

CT

Computed tomography.

RN

Radical nephrectomy.

RCC

Renal cell carcinoma.

UTUC

Upper urinary tract urothelial carcinoma.

EGFR

Epidermal growth factor receptor.

FISH

Fluorescence in situ hybridization.

RNU

Radical nephroureterectomy.

\section{Declarations}

\section{Ethics approval and consent to participate}

Written informed consent was obtained from the patient to participate the study. The study was approved by the ethics committee at SiChuan University.

\section{Consent for publication}

Written informed consent for publication of patient clinical details and/or clinical images was obtained from the patient. A copy of the consent form is available for review by the Editor of this journal.

\section{Availability of data and materials}

The datasets used and/or analysed during the current study are available from the corresponding authors on reasonable request. 


\section{Competing interests}

The authors declare that they have no competing interests.

\section{Funding}

This program was supported by the National Key Research and Development Program of China (Grant No. SQ2017YFSF090096), National Natural Science Foundation of China (Grant Nos. 81974098, 81770756, 81974099 and 81702536), programs from Science and Technology Department of Sichuan Province (Grant Nos. 2018JY0089, 2017HH0063 and 2018HH0153), Young Investigator Award of Sichuan University 2017 (Grant No. 2017SCU04A17).

\section{Authors contributions}

SJB, LL, and MS reviewed all literature available and wrote this manuscript. HX, HRL and WTZ provided medical care for the patient and collected the imaging data. LY and QW provided guidance throughout the process and fund. All authors contributed to data analysis, drafting or revising the article, gave final approval of the version to be published, and agree to be accountable for all aspects of the work.

\section{Acknowledgement}

Great appreciation should be given to Xiangyun Jian for her precious advice for this manuscript.

\section{References}

[1] Ferlay Jacques., Shin Hai-Rim., Bray Freddie., Forman David., Mathers Colin., Parkin Donald Maxwell. (2010). Estimates of worldwide burden of cancer in 2008: GLOBOCAN 2008. Int. J. Cancer, 127(12), 2893917. doi:10.1002/ijc.25516

[2] Ferlay J., Steliarova-Foucher E., Lortet-Tieulent J., Rosso S., Coebergh J W W., Comber H., Forman D., Bray F.(2013). Cancer incidence and mortality patterns in Europe: estimates for 40 countries in 2012. Eur. J. Cancer, 49(6), 1374-403. doi:10.1016/j.ejca.2012.12.027

[3] Jemal Ahmedin., Bray Freddie., Center Melissa M., Ferlay Jacques., Ward Elizabeth., Forman David. (2011). Global cancer statistics. CA Cancer J Clin, 61(2), 69-90. doi:10.3322/caac.20107

[4] AIOM,AIRTUM. I Numeri Del Cancro in Italia 2018. Milan:AIMO;2018

[5] Pouliot Frédéric., Shuch Brian., Larochelle Jeffrey C., Pantuck Allan., Belldegrun Arie S.(2010). Contemporary management of renal tumors with venous tumor thrombus. J. Urol., 184(3), 833-41; quiz 1235. doi:10.1016/j.juro.2010.04.071 
[6] Kim Hyung L., Zisman Amnon., Han Ken-Ryu., Figlin Robert A., Belldegrun Arie S.(2004). Prognostic significance of venous thrombus in renal cell carcinoma. Are renal vein and inferior vena cava involvement different? J. Urol., 171(null), 588-91. doi:10.1097/01.ju.0000104672.37029.4b

[7] Hartman D S., Pyatt R S., Dailey E.(1983). Transitional cell carcinoma of the kidney with invasion into the renal vein. Urol Radiol, 5(2), 83-7. doi:10.1007/BF02926776

[8] Huber Johannes., Teber Dogu., Hatiboglu Gencay., Popeneciu Valentin., Jakobi Hildegard., Hallscheidt Peter., Pahernik Sascha., Hohenfellner Markus.(2014). Does a venous tumor thrombus exclude renal transitional cell carcinoma? Implications for neo-adjuvant treatment strategies. Anticancer Res., 34(2), 1031-5

[9] Messer Jamie., Shariat Shahrokh F., Brien James C., Herman Michael P., Ng Casey K., Scherr Douglas S., Scoll Benjamin., Uzzo Robert G., Wille Mark., Eggener Scott E., Steinberg Gary., Terrell John D., Lucas Steven M., Lotan Yair., Boorjian Stephen A., Raman Jay D.(2011). Urinary cytology has a poor performance for predicting invasive or high-grade upper-tract urothelial carcinoma. BJU Int., 108(5), 7015. doi:10.1111/j.1464-410X.2010.09899.x

[10] Voulgaris E., Pentheroudakis G., Pappa L., Bafa M., Goussia A., Dalezis P., Tsombanidou C., Geromichalos G., Papageorgiou A., Koutsilieris M., Malamou-Mitsi V., Pavlidis N.(2011). Positive urinary cytology in patients with lung cancer in the absence of obvious urine tract metastases. Lung Cancer, 73(1), 51-8. doi:10.1016/j.lungcan.2010.10.018

[11] Rouprêt M, et al. European Association of Urology Guidelines on Upper Urinary Tract Urothelial Carcinoma: 2017 Update. Eur Urol (2017), http://dx.doi.org/10.1016/j.eururo.2017.07.036

[12] Baard Joyce., Freund Jan Erik., de la Rosette Jean J M C H., Laguna Maria P.(2017). New technologies for upper tract urothelial carcinoma management. Curr Opin Urol, 27(2), 170-175. doi:10.1097/MOU.0000000000000373

\section{Figures}




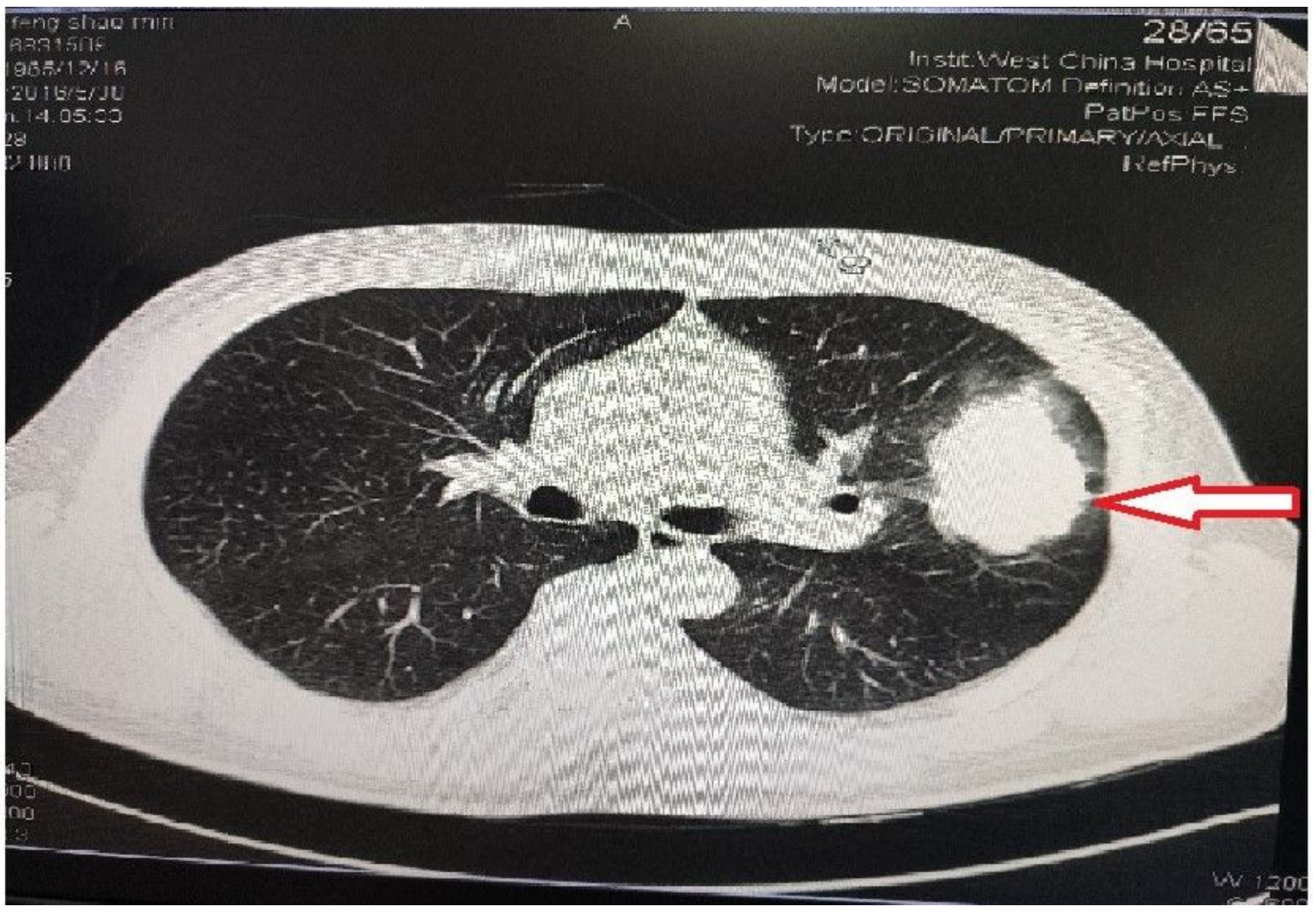

Figure 1

CT scan of chest showed a mass in the left upper lung in June 2016.

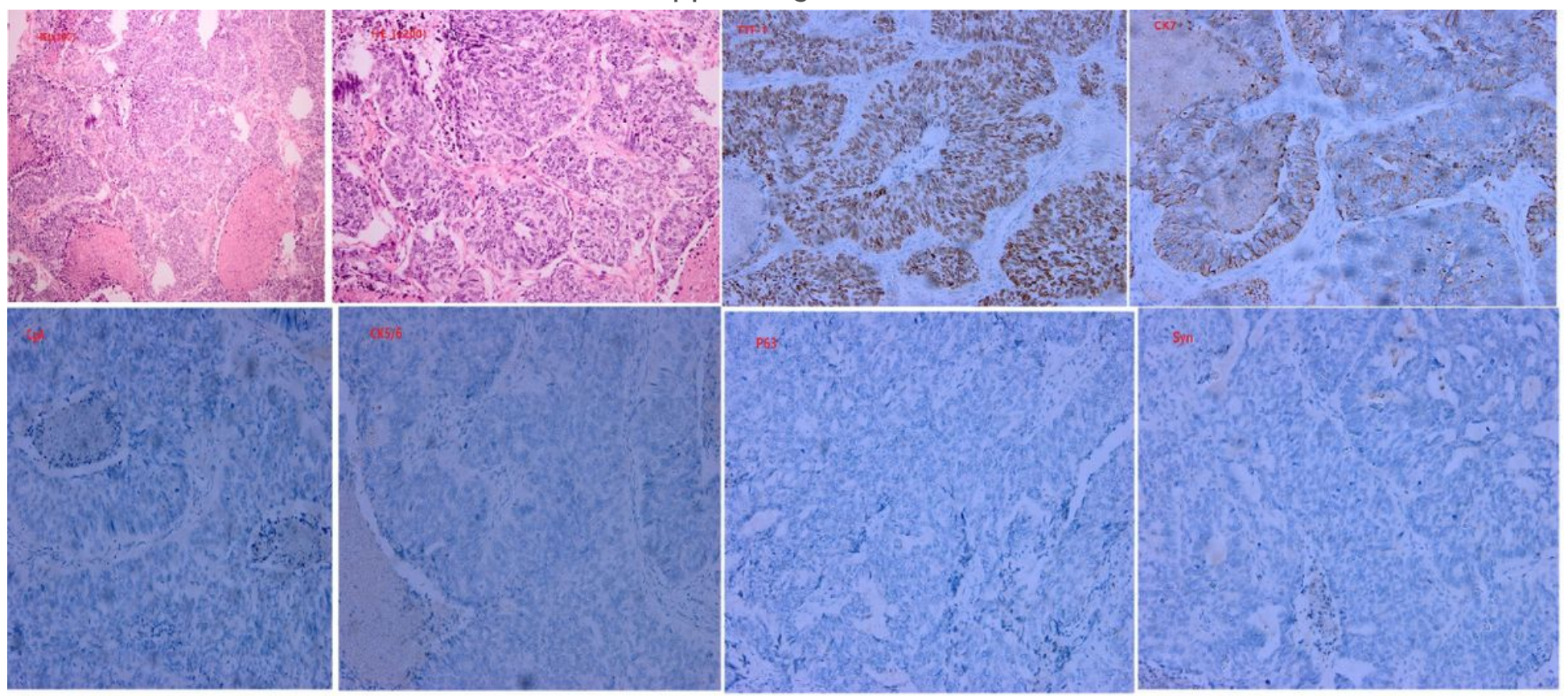

Figure 2 
HE staining (x100 and x200) of lung adenocarcinoma classified between poorly differentiated and moderately differentiated. Immunohistochemistry staining (x200) of the mass in the left upper lobe. The expression of TTF-1, CK7 were positive, while the expressions of P63, CK5/6, Syn and CgA were negative.

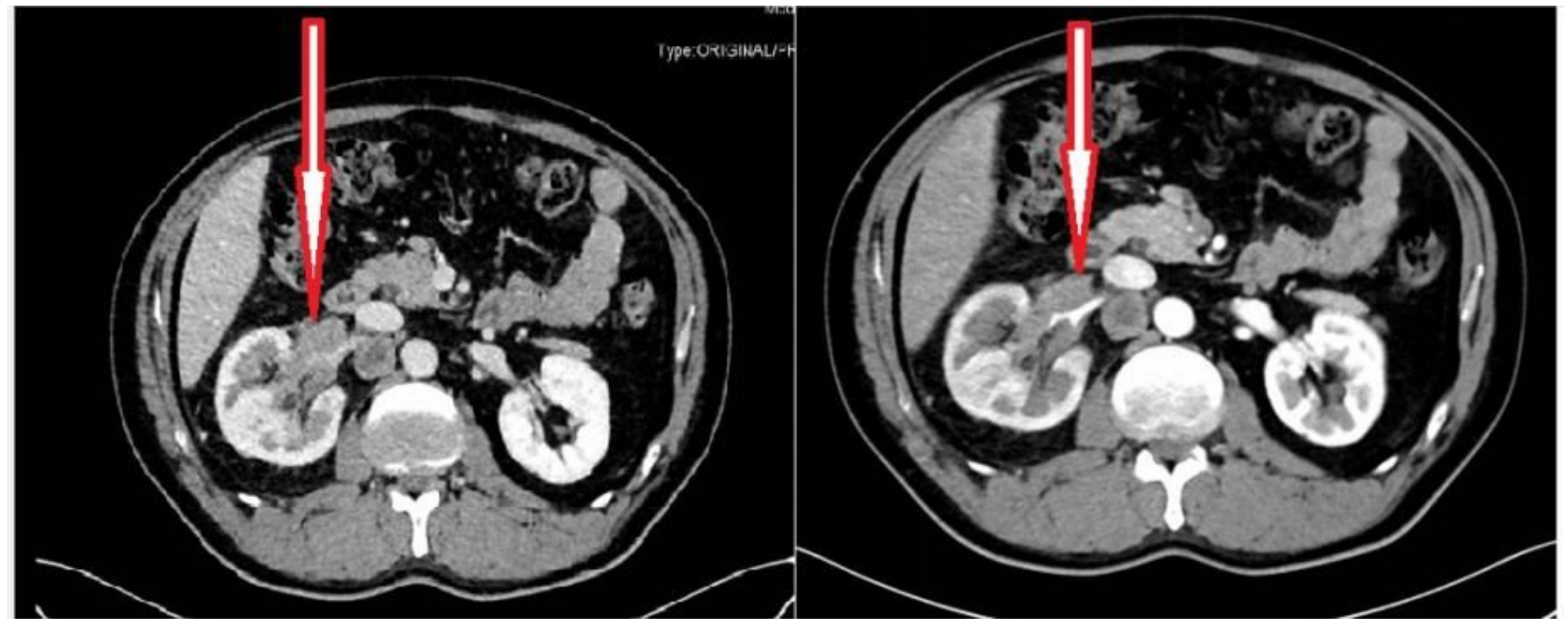

\section{Figure 3}

Contrast enhanced computed tomography scan of the abdomen revealed a neoplasm in the right renal pelvis, and filling coloboma could be seen in the right renal vein.
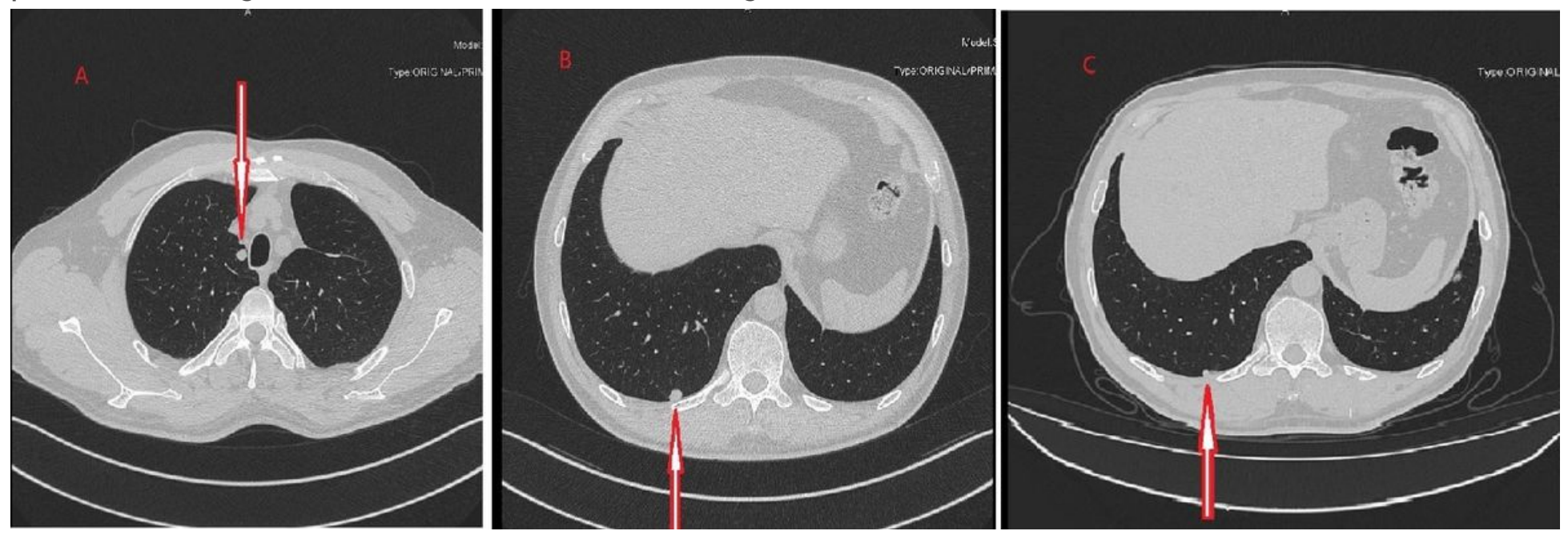

Figure 4

A and B: CT scan of chest showed two nodules in his right lung in December 2017. C: CT scan of chest in March 2020 showed the nodule located in the right inferior lobe has experienced a significant decrease, nodal diameter changed from $10 \mathrm{~mm}$ to $3.7 \mathrm{~mm}$. 


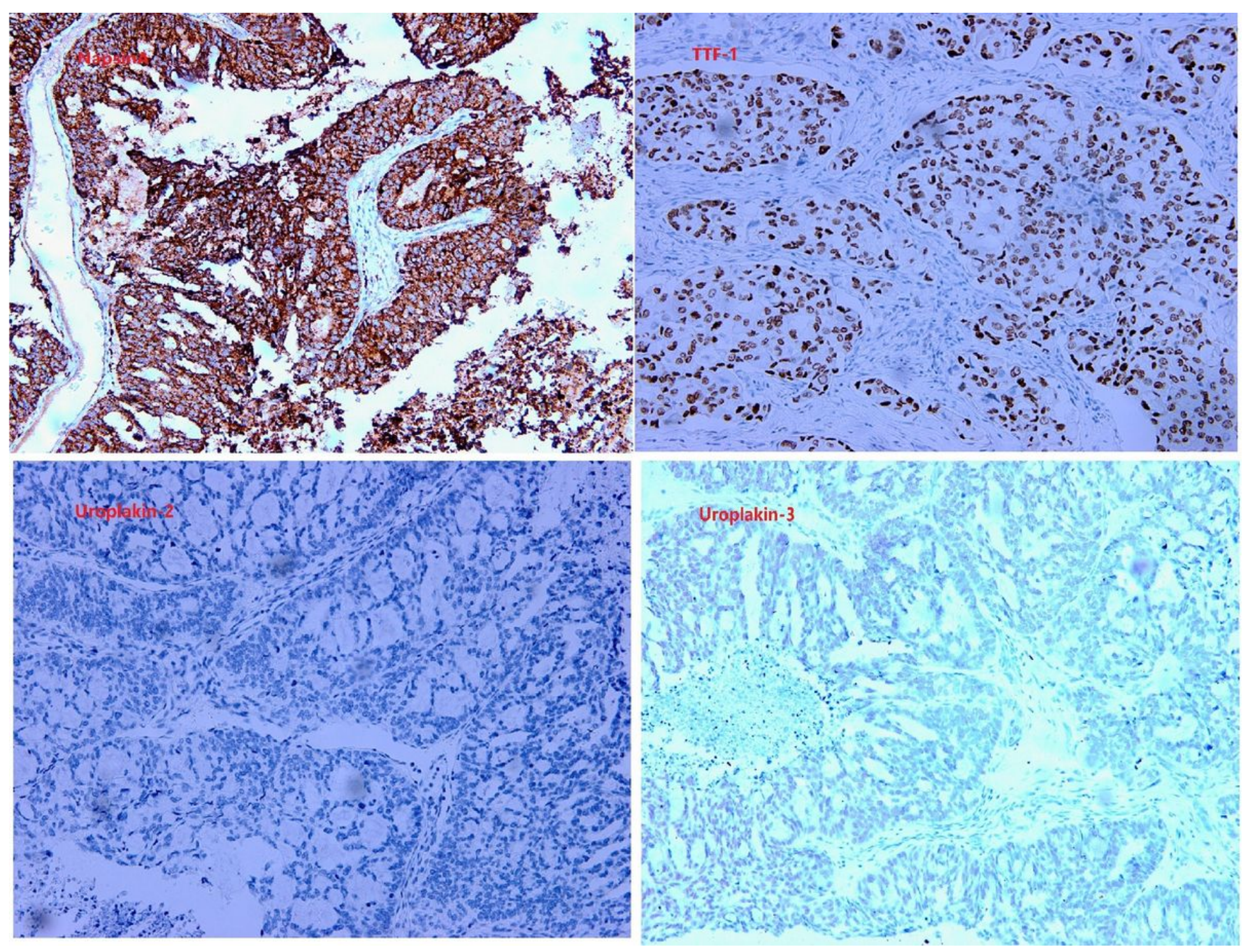

\section{Figure 5}

Immunohistochemistry staining (x200) of the neoplasm occupying the right pelvis and the tumor thrombus. Positive for TTF-1, NapsinA and negative for Uroplakin-2, Uroplakin-3. 